\title{
SatGen - II. Assessing the impact of a disc potential on subhalo populations
}

\author{
Sheridan B. Green ${ }^{\circledR},{ }^{1 \star} \dagger$ Frank C. van den Bosch ${ }^{\circledR 1,2}$ and Fangzhou Jiang ${ }^{\circledR 3,4} \ddagger$ \\ ${ }^{1}$ Department of Physics, Yale University, PO Box 208120, New Haven, CT 06520-8120, USA \\ ${ }^{2}$ Department of Astronomy, Yale University, PO Box 208101, New Haven, CT 06520-8101, USA \\ ${ }^{3}$ TAPIR, California Institute of Technology, Pasadena, CA 91125, USA \\ ${ }^{4}$ Carnegie Observatories, 813 Santa Barbara Street, Pasadena, CA 91101, USA
}

Accepted 2021 October 22. Received 2021 September 17; in original form 2021 May 13

\begin{abstract}
The demographics of dark matter substructure depend sensitively on the nature of dark matter. Optimally leveraging this probe requires accurate theoretical predictions regarding the abundance of subhaloes. These predictions are hampered by artificial disruption in numerical simulations, by large halo-to-halo variance, and by the fact that the results depend on the baryonic physics of galaxy formation. In particular, numerical simulations have shown that the formation of a central disc can drastically reduce the abundance of substructure compared to a dark matter-only simulation, which has been attributed to enhanced destruction of substructure due to disc shocking. We examine the impact of discs on substructure using the semi-analytical subhalo model SatGen, which accurately models the tidal evolution of substructure free of the numerical disruption that still hampers $N$-body simulations. Using a sample of 10000 merger trees of Milky Way-like haloes, we study the demographics of subhaloes that are evolved under a range of composite halo-disc potentials with unprecedented statistical power. We find that the overall subhalo abundance is relatively insensitive to properties of the disc aside from its total mass. For a disc that contains 5 per cent of $M_{\text {vir }}$, the mean subhalo abundance within $r_{\text {vir }}$ is suppressed by $\lesssim 10$ per cent relative to the no-disc case, a difference that is dwarfed by halo-to-halo variance. For the same disc mass, the abundance of subhaloes within $50 \mathrm{kpc}$ is reduced by $\sim 30$ per cent. We argue that the disc mainly drives excess mass-loss for subhaloes with small pericentric radii and that the impact of disc shocking is negligible.
\end{abstract}

Key words: methods: numerical-galaxies: haloes - dark matter.

\section{INTRODUCTION}

The substructure present in dark matter (DM) haloes is the outcome of a hierarchical assembly process combined with tidal and impulsive forces that work to dissolve it. Since the particle nature of DM impacts the mass function and density profiles of DM haloes, it also affects the demographics of its substructure. For example, if DM is 'warm', the abundance of low-mass subhaloes is suppressed relative to that which is predicted for cold dark matter (e.g. Lovell et al. 2014; Bose et al. 2017). If DM undergoes significant self-interaction, or is an ultralight boson, the inner halo density profile becomes cored (e.g. Kaplinghat, Tulin \& Yu 2016; Robles et al. 2017; Burkert 2020) and is less resilient to tidal forces (Peñarrubia et al. 2010), resulting in an overall suppression of substructure. This powerful potential to place constraints on DM has prompted various observational attempts to quantify the abundance of DM substructure, including searches for gaps in stellar streams (e.g. Erkal et al. 2016; Bonaca et al. 2020; Banik et al. 2021), measurements of gravitational lensing distortions (e.g. Vegetti et al. 2014; Hezaveh et al. 2016; Nierenberg et al. 2017), indirect detection studies that search for DM annihilation signals (e.g.

\footnotetext{
*E-mail: sheridan.green@yale.edu $\dagger$ NSF Graduate Research Fellow.

$\ddagger$ Troesh Scholar.
}

Stref \& Lavalle 2017; Somalwar et al. 2021), and measurements of the abundance of satellite galaxies (via the galaxy-halo connection; e.g. Nadler et al. 2021).

In order to fully leverage these observations to constrain DM microphysics, it is prudent that we are able to accurately predict subhalo abundances for the different DM models. Arguably, the best way to account for all the relevant, strongly non-linear physical processes is to use full cosmo-hydrodynamical simulations of galaxy formation (e.g. Wetzel et al. 2016; Pillepich et al. 2018) at a resolution sufficient to resolve substructure in the relevant mass range. Unfortunately, the computationally demanding nature of such simulations as well as the uncertainties related to sub-grid physics modelling represent significant roadblocks for their use in such a task. As a consequence, DM-only cosmological simulations (e.g. Springel et al. 2008; Klypin, Trujillo-Gomez \& Primack 2011) are typically used as a less expensive alternative. However, these simulationbased approaches are still adversely impacted by artificial subhalo disruption and limited mass resolution (van den Bosch 2017; van den Bosch \& Ogiya 2018; Green, van den Bosch \& Jiang 2021). Semi-analytical models (SAMs; e.g. Taylor \& Babul 2004; Zentner et al. 2005a; Jiang \& van den Bosch 2016; Jiang et al. 2021) provide attractive alternatives for predicting the substructure abundance in a manner that is both computationally efficient and insensitive to the particular numerical limitations of $N$-body simulations. 
Unfortunately, DM-only simulations and SAMs typically do not account for the impact of baryons on the subhalo population, which can be quite important. For example, the aforementioned observational probes are most sensitive to the inner halo, where the central galaxy significantly influences the host potential. Several studies have demonstrated that a central galactic disc suppresses the overall subhalo abundance. For example, D’Onghia et al. (2010) grew an analytical disc potential in a high-resolution cosmological zoom-in simulation of a Milky Way-like (MW) halo and showed that substructure in the inner regions of the halo is efficiently destroyed, which they ascribed to disc shocking. More recently, GarrisonKimmel et al. (2017) found that the suppression in subhalo abundance seen in a full physics simulation relative to a DM-only realization of the same halo can be reproduced by simply embedding a disc potential within the DM-only halo. Both Peñarrubia et al. (2010) and Errani et al. (2017) used idealized simulations to examine the impact of a central disc on the abundance of subhaloes, confirming once more that the presence of a disc can significantly deplete the subhalo population, especially towards the centre of the halo.

Since a disc potential drives additional subhalo mass-loss, its presence must be properly accounted for in any successful substructure modelling endeavour. However, to date, no study has been able to assess the impact of the disc on subhalo populations in a statistically meaningful way. Recently, we introduced SatGen (Jiang et al. 2021), a SAM framework that can rapidly generate random substructure realizations, thereby enabling a robust treatment of the halo-to-halo variance. As shown in Jiang \& van den Bosch (2017), this variance can be very large and is strongly correlated with the formation time and concentration of the host halo (see also Zentner et al. 2005a; Giocoli et al. 2010). Furthermore, SatGen can be used to isolate the influence of the disc from assembly history variation by studying how subhaloes from the same merger tree evolve under different host potentials. Due to its speed, SatGen is also ideal for assessing how sensitive the subhalo statistics are to parameters of the disc model via sweeps of the parameter space.

In this paper, we use SatGen to investigate the differential impact of a galactic disc potential on the subhalo populations of Milky Way-like haloes. We initially explored the influence of a disc in Jiang et al. (2021) - here, we build upon this pilot study by greatly boosting the size of our halo sample, exploring a wide range of disc models, and incorporating a more sophisticated subhalo tidal evolution model. While our findings are in good agreement with the simulation results of Errani et al. (2017) and Garrison-Kimmel et al. (2017), our ability to study a large halo sample and, thus, probe the halo-to-halo variance sheds new light on the statistical relevance of these results. We track individual subhaloes and illustrate how their masses are altered due to an embedded central disc. We also search for the presence of a disc-driven angular bias in the spatial distribution of subhaloes, as well as show that the overall subhalo abundance is relatively insensitive to the size and growth history of the disc and is only affected by the disc mass. This paper is organized as follows. In Section 2, we first provide an overview of our semi-analytical modelling framework. The results are presented in Section 3, which is followed by a detailed discussion (Section 4) as to whether 'disc shocking' or enhanced tidal stripping serves as the dominant disc-driven subhalo depletion mechanism. Finally, in Section 5, we summarize our findings and motivate future work.

Throughout this work, the halo mass is defined as the mass enclosed within the virial radius, $r_{\text {vir }}$, inside of which the mean density is equal to $\Delta_{\text {vir }}(z)$ times the critical density. For the $\Lambda$ cold dark matter $(\Lambda \mathrm{CDM})$ cosmology that we adopt $\left(h=0.7, \Omega_{\mathrm{m}}=0.3\right.$, $\left.\Omega_{\Lambda}=0.7, \Omega_{\mathrm{b}}=0.0465, \sigma_{8}=0.8, n_{\mathrm{s}}=1.0\right), \Delta_{\mathrm{vir}}(z=0) \approx 100$ and is otherwise well-described by the fitting formula presented by Bryan \& Norman (1998). Throughout, we use $m$ and $M$ to denote subhalo and host halo masses, respectively. We use $l$ and $r$ to reference subhaloand host halocentric radii, respectively. The base-10 logarithm is denoted by $\log$ and the natural logarithm is denoted by $\mathrm{ln}$.

\section{SEMI-ANALYTICAL METHODS}

This study employs the SatGen semi-analytical modelling framework that is presented by Jiang et al. (2021). In particular, we use the model of subhalo tidal evolution recently developed by Green \& van den Bosch (2019) and Green et al. (2021). This model has been calibrated using the Dynamical Aspects of Subhaloes idealized simulation library (hereafter $D A S H$; Ogiya et al. 2019) to accurately reproduce the bound mass and density profiles of simulated $\mathrm{N}$ body subhaloes as they orbit within an analytical host potential. We refer the reader to these papers for a comprehensive description of the model. In short, SatGen combines prescriptions for (i) analytical halo merger trees (Parkinson, Cole \& Helly 2008), (ii) subhalo orbit initialization (Li et al. 2020), (iii) orbit integration, including dynamical friction (Chandrasekhar 1943), (iv) density profile evolution (Green \& van den Bosch 2019), and (v) tidal massloss (Green et al. 2021) in order to generate subhalo catalogues (which include both mass and position information) for ensembles of host halo realizations.

Both the host halo and the initial subhaloes (i.e. at infall) are assumed to have Navarro, Frenk \& White (1997, hereafter NFW) density profiles with concentrations computed via the model of Zhao et al. (2009). Each subhalo is integrated along its orbit and experiences tidal mass-loss, which is given by

$$
\frac{\Delta m}{\Delta t}=-\alpha \frac{m\left(>l_{\mathrm{t}}\right)}{t_{\mathrm{char}}} \text {. }
$$

Here, $t_{\text {char }}$ is the characteristic orbital time of the subhalo, $m(>l)$ is the subhalo mass that lies outside of radius $l$, with $l_{\mathrm{t}}$ denoting the instantaneous tidal radius (defined below), and $\alpha$ is a calibrated parameter that controls the stripping efficiency. Motivated by the work of Hayashi et al. (2003) and Peñarrubia, Navarro \& McConnachie (2008), the density profiles of stripped subhaloes are modelled according to

$\rho(l, t)=H\left(l \mid f_{\mathrm{b}}(t), c_{\mathrm{vir}, \mathrm{s}}\right) \rho\left(l, t_{\mathrm{acc}}\right)$,

where $f_{\mathrm{b}}(t)$ is the bound mass fraction of the subhalo, $c_{\mathrm{vir}, \mathrm{s}}$ is the concentration of the subhalo at accretion, and $t_{\text {acc }}$ denotes the time of accretion. For the 'transfer function', $H(l)$, we use the model of Green \& van den Bosch (2019), which has been carefully calibrated against the $D A S H$ simulations.

The galactic disc, when included, is positioned at the centre of the host halo and modelled with the axisymmetric Miyamoto \& Nagai (1975, hereafter MN) density profile, which has three parameters: (i) the radial scale length, $a_{\mathrm{d}}$, (ii) vertical scale height, $b_{\mathrm{d}}$, and (iii) mass, $M_{\mathrm{d}}$. We write $a_{\mathrm{d}}=f_{a}\left[M_{\mathrm{vir}}(z) / M_{0}\right]^{\beta_{a}} r_{\mathrm{vir}, 0}, M_{\mathrm{d}}=$ $f_{M}\left[M_{\mathrm{vir}}(z) / M_{0}\right]^{\beta_{M}} M_{0}$, and set $b_{\mathrm{d}}$ to be a fixed fraction of $a_{\mathrm{d}}$. Here, $M_{\mathrm{vir}}(z)$ is the mass accretion history of the host halo, $M_{0}=M_{\mathrm{vir}}(z=$ 0 ), and $r_{\mathrm{vir}, 0}$ is the virial radius of the host at $z=0$. In this work, we restrict ourselves to host haloes that reach a virial mass of $M_{0}=10^{12} h^{-1} \mathrm{M}_{\odot}$ at $z=0$, which corresponds to $r_{\mathrm{vir}, 0} \approx 290 \mathrm{kpc}$. Our fiducial, Milky Way-like disc is described by $f_{a}=0.0125, b_{\mathrm{d}} / a_{\mathrm{d}}=$ $0.08, \beta_{a}=1 / 3, f_{M}=0.05$, and $\beta_{M}=1$, such that the disc mass grows linearly with $M_{\mathrm{vir}}(z)$ and the scale length grows linearly with $r_{\text {vir }}(z)$, in good agreement with both empirical constraints (Kravtsov 2013) and simulation results (Jiang et al. 2019). The disc properties at $z=0$ are $a_{\mathrm{d}} \approx 3.6 \mathrm{kpc}, b_{\mathrm{d}} \approx 0.3 \mathrm{kpc}$ (i.e. a relatively thin 
disc), and $M_{\mathrm{d}}=5 \times 10^{10} h^{-1} \mathrm{M}_{\odot}$, reminiscent of the Milky Way. The parametrization chosen is sufficiently flexible to enable us to study the impact of the disc growth history and structure on the subhalo population by simply varying $f_{a}, \beta_{a}, f_{M}, \beta_{M}$, and $b_{\mathrm{d}} / a_{\mathrm{d}}$. As we show in Section 3.5, though, the results are relatively insensitive to all but the final disc mass $\left(f_{M}\right)$. We emphasize that the total mass of the halo-disc system enclosed within $r_{\text {vir }}(z)$ sums to $M_{\text {vir }}(z)$. In order to achieve this, we simply multiply the host NFW density profile by $\left[M_{\mathrm{vir}}(z)-M_{\mathrm{d}}(z)\right] / M_{\mathrm{vir}}(z)$.

The merger tree resolution, which sets the lower limit on the subhalo accretion mass, corresponds to $m_{\text {res }} \geq 10^{8} h^{-1} \mathrm{M}_{\odot}$. All subhaloes are evolved until their mass falls below $m=10^{-5} m_{\text {acc }}$. All results are averaged over 10000 host halo merger tree realizations. Specifically, we use the same set of merger trees throughout in order to isolate the differential impact of the disc from the effect of assembly history variation.

In order to strengthen the performance of our mass-loss model with composite halo-disc hosts, we make two changes to SatGen relative to its specification in Jiang et al. (2021) and Green et al. (2021). Since we are working with an axisymmetric potential, we make the following substitution for the tidal radius definition:

$l_{\mathrm{t}}=\left[\frac{m\left(<l_{\mathrm{t}}\right) / M(<r)}{2+\frac{\Omega^{2} r^{3}}{G M(<r)}-\left.\frac{\mathrm{d} \ln M}{\mathrm{~d} \ln r}\right|_{r}}\right]^{1 / 3} \Rightarrow l_{\mathrm{t}}=\left[\frac{G m\left(<l_{\mathrm{t}}\right)}{\Omega^{2}-\left.\frac{\mathrm{d}^{2} \Phi}{\mathrm{d} r^{2}}\right|_{r}}\right]^{1 / 3}$.

Here, $\Omega$ is the instantaneous angular velocity of the subhalo and $\Phi$ is the gravitational potential of the host halo-disc system. Note that these two definitions of $l_{t}$, introduced by King (1962), are identical when the host potential is spherically symmetric. However, the definition on the right is more general, as it does not depend on spherical averages of the host properties. To wit, the radial derivative of an axisymmetric potential is $\frac{\mathrm{d} \Phi}{\mathrm{d} r}=\frac{\partial \Phi}{\partial R} \frac{\mathrm{d} R}{\mathrm{~d} r}+\frac{\partial \Phi}{\partial z} \frac{\mathrm{d} z}{\mathrm{~d} r}$. Hence, for spherical hosts, the mass-loss model of equation (1), which was calibrated using the definition on the left, remains unchanged after this substitution. The second change is with regards to the mass-loss coefficient, $\alpha$, which is a function of the ratio between the host and subhalo concentrations (see Green et al. 2021). In order to account for the modified mass distribution in the presence of a disc, we modify the host concentration used to compute $\alpha$ according to the following procedure. We define a new scale radius, $r_{\mathrm{s}, \mathrm{d}}$, as the radius within which the enclosed mass of the combined halo-disc system is the same as that which is enclosed within the scale radius of the NFW halo-only host. The modified host concentration is simply $r_{\mathrm{vir}} / r_{\mathrm{s}, \mathrm{d}}$, which is somewhat larger than the halo-only concentration due to the compactness of the disc. Note that, unlike Jiang et al. (2021), we omit the effect of adiabatic contraction on the host concentration since we are interested in studying relative disc-driven subhalo depletion. However, we emphasize that a baryon-driven increase in the DM concentration is degenerate with an increased disc mass, which we demonstrate in Section 3.6.

Since we calibrated our mass-loss model using the DASH simulations, which only include spherical NFW host haloes, we must verify that the prescription remains valid for subhaloes that evolve in the combined presence of an NFW halo and an MN disc. To this end, we run an additional set of idealized simulations and compare the predictions of our mass-loss model (with the substituted $l_{\mathrm{t}}$ definition and modified host concentration definition) to the mass trajectories of the simulated subhaloes. The simulation methods and model comparison results are described in Appendix A. In summary, we find that, after making the two aforementioned modifications, the model generalizes well to combined halo-disc potentials and, thus, we proceed to use this modified version of SatGen in this work.

\section{RESULTS}

\subsection{Subhalo mass functions}

We begin by assessing the impact of the disc on the abundance of subhaloes as a function of their mass. Fig. 1 plots the cumulative subhalo mass functions (SHMF), $N\left(>m / M_{0}\right)$, for the disc-less case (blue lines) as well as for several disc configurations, each with our fiducial $f_{a}, \beta_{a}$, and $\beta_{M}$, but with different values of the disc mass fraction, $f_{M}$, as indicated. The range of disc mass fractions covered $\left(f_{M} \in[0.025,0.05,0.075,0.1]\right)$ is motivated by estimates of the stellar mass-halo mass relation (see e.g. Moster et al. 2010; More et al. 2011; Behroozi et al. 2019). The dark and light shaded regions denote the 16-84 and 2.5-97.5 percentile intervals, respectively, of the individual SHMFs in the halo-only (i.e. no-disc) case, highlighting the typical halo-to-halo variance. The SHMFs in the left-hand panel include subhaloes of all orders ${ }^{1}$ that have an instantaneous hostcentric radius at $z=0$ of $r<r_{\mathrm{vir}, 0}$. In order to emphasize the pronounced effect of the disc in the halo centre, the SHMFs in the right-hand panel are restricted to subhaloes with $r<50 \mathrm{kpc}$. Clearly, the disc results in a suppression of the subhalo abundance that is proportional to the disc mass fraction, $f_{M}$. However, the halo-tohalo variance in the SHMF is dramatically larger than the difference between the mean SHMFs from the various disc configurations (this finding is also present throughout the results of Jiang et al. 2021). The most massive disc, with $f_{M}=0.1$, results in a $\sim 0.09$ dex (18 per cent) suppression in the $r<r_{\mathrm{vir}, 0}$ SHMF at the low-mass end, which decreases slightly with increasing $m / M_{0}$. Our results are in excellent agreement with the idealized simulations of Errani et al. (2017), who report a 20 per cent suppression in the SHMF of a cuspy Milky Waymass halo due to the presence of a disc with $f_{M}=0.1$ (note that they also find the effect to be reduced at the high-mass end of the SHMF). The factor of suppression due to the disc is greatly increased when we restrict the SHMF to subhaloes with $r<50 \mathrm{kpc}$. For example, the $f_{M}=0.1$ disc drives a $\sim 0.25 \operatorname{dex}(\sim 44$ per cent $)$ decrease in subhaloes within $50 \mathrm{kpc}$, which is consistent with D'Onghia et al. (2010). Our mean results are also in excellent agreement with Jiang et al. (2021), indicating an overall insensitivity to our differing subhalo tidal evolution models. ${ }^{2}$

We find that the slope of the $r<r_{\text {vir, } 0}$ SHMF changes very little with $f_{M}$, ranging from -0.91 in the disc-less case to -0.89 in the $f_{M}=$ 0.1 configuration. The same is true of the $r<50 \mathrm{kpc}$ SHMF, which has a slope of -0.81 in the disc-less case and -0.79 when $f_{M}=0.1$. Taken together, these results imply that mass segregation (or the lack thereof, cf. van den Bosch et al. 2016) is not greatly impacted by the disc. However, because of the slight $f_{M}$-dependence of the slope, the $r$ $<r_{\text {vir, } 0}$ SHMF residuals exhibit a small amount of mass-dependence, especially for the larger $f_{M}$. In order to gauge how this plays out at the low mass end (i.e. $\log \left(m / M_{0}\right)<-4$ ), we generated 2000 merger trees with enhanced resolution $\left(m_{\text {acc }} \geq 10^{7} h^{-1} \mathrm{M}_{\odot}\right)$ and evolved the subhaloes using each of the five configurations introduced in Fig. 1. We report that the residuals for $10^{-5} \leq m / M_{0} \leq 10^{-4}$ flatten off and are consistent with their values at $m / M_{0}=10^{-4}$. This convergence of the slopes in the low- $m / M_{0}$ limit indicates that the additional dynamical friction due to the disc, which only impacts more massive

\footnotetext{
${ }^{1}$ A subhalo of order $n$ is hosted by a (sub)halo of order $n-1$, with host haloes corresponding to order 0 .

${ }^{2}$ Note that Jiang et al. (2021) also account for the fact that the subhalo density profile may be affected by baryons prior to infall. However, this only affects the small fraction of subhaloes that host bright satellites, the analysis of which is beyond the scope of this study.
} 

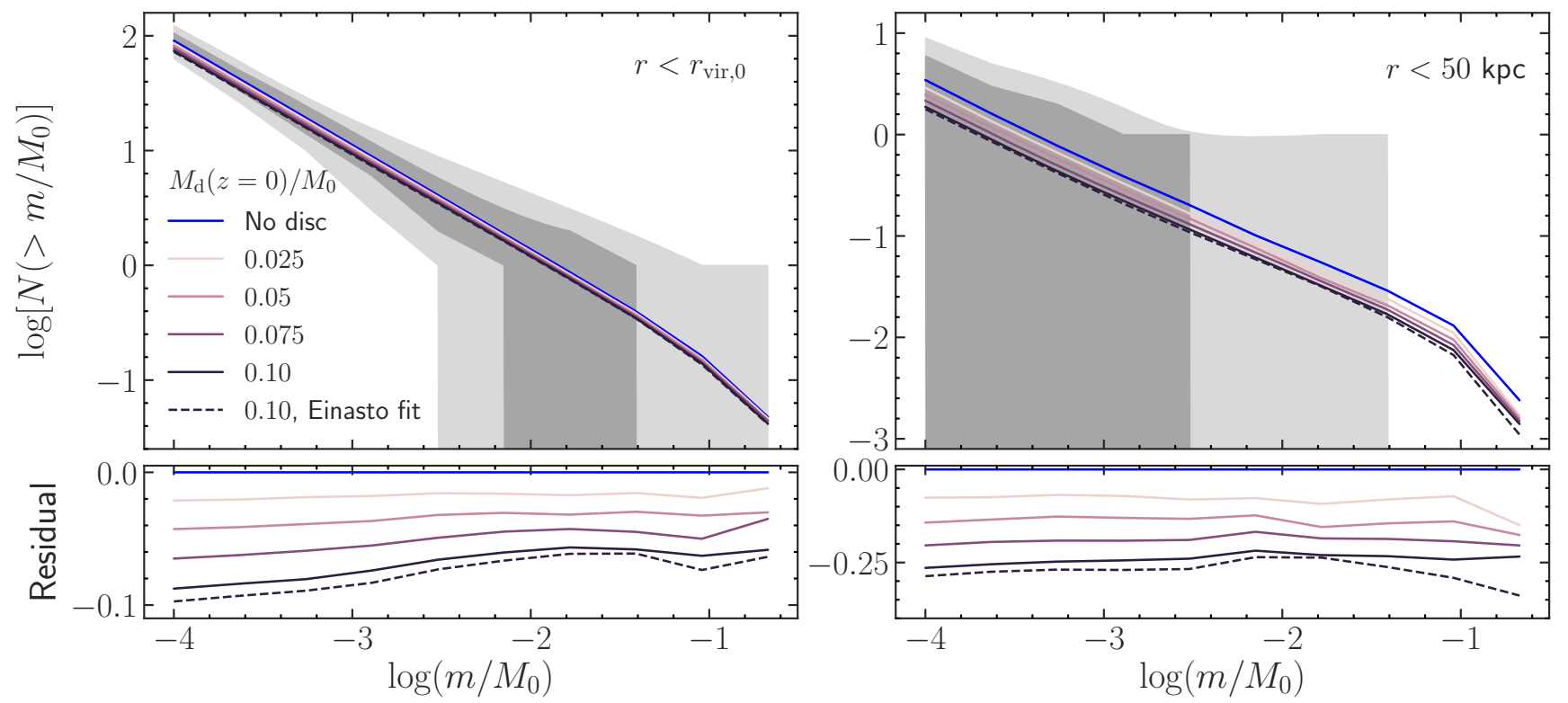

Figure 1. The subhalo mass function, which includes subhaloes of all orders with a $z=0$ host-centric radius of (left-hand panel) $r<r_{\mathrm{vir}, 0}$ or (right-hand panel) $r<50 \mathrm{kpc}$. The curves denote the mean SHMF taken over 10000 trees whereas the dark and light shaded regions correspond to the $16-84$ and $2.5-97.5$ percentiles, respectively, of the individual (halo-only) trees. The different curves illustrate the dependence of the final disc mass on the subhalo population. The suppression of the subhalo abundance is proportional to the disc mass and is much larger in the halo centre. The dashed lines correspond to the replacement of the $f_{M}=0.1$ disc with an Einasto (1965) sphere that has a nearly equivalent enclosed mass profile. The agreement between these dashed lines and the corresponding $f_{M}=0.1$ disc curves demonstrates the insignificance of 'disc shocking'.

subhaloes, is the most likely cause of the minor $f_{M}$-dependence of the SHMF slope.

In order to aid our evaluation of the significance of 'disc shocking', we consider a case where the MN disc is replaced by a spherical component with a nearly equivalent spherically enclosed mass profile. Specifically, we fit the $M(<r)$ of an Einasto (1965) profile to the $M(<r)$ of an MN disc with $b_{\mathrm{d}} / a_{\mathrm{d}}=0.08$. Using the notation of Jiang et al. (2021, Appendix A3), the parameters of the resultant Einasto (1965) halo are $M_{\mathrm{tot}}=M_{\mathrm{d}}$ (i.e. the total mass of the system is unchanged), $n=2.13$, and $c_{2}=43.7$. The concentration is defined with respect to the $z=0$ virial mass definition. Using this convention, the mass and size of the spherical substitute grow identically to that of the disc by simply holding $M_{\mathrm{tot}}=M_{\mathrm{d}}$. The Einasto (1965) sphere is slightly more centrally concentrated - its enclosed mass is $\sim 12$ per cent larger than that of the disc at $r \approx 6 a_{\mathrm{d}}$, with the $M(<r)$ of the two systems converging as $r$ increases. We evolve the subhaloes in this composite halo-sphere host with $f_{M}=0.1$, presenting the resulting SHMF as dashed lines in Fig. 1. The results agree quite well with those of the $f_{M}=0.1$ disc. The slightly increased central concentration of the spherical replacement appears to drive a minor increase in the overall mass-loss relative to the disc. This agreement between the impact of a disc and a spherical replacement is also reported by Garrison-Kimmel et al. (2017). We elaborate on the implications of this finding in Section 4.

\subsection{Radial profiles}

In Fig. 2, we shift our attention to the cumulative radial subhalo abundance profile, $N\left(<r / r_{\text {vir, } 0}\right)$. Here, we restrict ourselves to two different subsets of the subhalo population. In the left-hand panel, we only count subhaloes that have a maximum circular velocity at accretion, $V_{\max , \text { acc }}$, that is greater than $30 \mathrm{~km} \mathrm{~s}^{-1}$, which roughly captures the population of subhaloes that could themselves host galaxies. We note that all such subhaloes with $\mathrm{m} / \mathrm{m}_{\text {acc }}>10^{-5}$ are included; however, we find that the results presented throughout this work are qualitatively insensitive to the choice of $\mathrm{m} / \mathrm{m}_{\text {acc }}$ used as the cut-off for inclusion. In the right-hand panel, we instead include all subhaloes with instantaneous mass at $z=0$ of $m>10^{8} h^{-1} \mathrm{M}_{\odot}$. Once again, the dark and light shaded regions indicate the 68 per cent and 95 percent halo-to-halo variance intervals in the no-disc case. The mean profile of each disc configuration is again enclosed within the halo-to-halo variance of the disc-less profile. The radial profiles further illustrate the enhanced impact of the disc on subhalo statistics towards the halo centre, as the mean profiles are increasingly suppressed with decreasing $r / r_{\text {vir, } 0}$. This effect is strongest on the population with $V_{\max \text {, acc }}>30 \mathrm{~km} \mathrm{~s}^{-1}$, which is reduced by roughly $0.7 \mathrm{dex}(\approx 80$ per cent $)$ within $20 \mathrm{kpc}$ of the halo centre when $f_{M}=0.1$. In comparison to all subhaloes with $m>10^{8} h^{-1} \mathrm{M}_{\odot}$, the population with $V_{\max \text {, acc }}>30 \mathrm{~km} \mathrm{~s}^{-1}$ is composed of a larger fraction of initially massive subhaloes, which experience stronger dynamical friction and, thus, enhanced tidal stripping. We find that the fractional impact of the disc on the radial profiles of the two simulated systems studied by Garrison-Kimmel et al. (2017) is within the halo-to-halo variance of our log-residuals (see also Jiang et al. 2021). Once again, the replacement of the disc with a nearly equivalent Einasto (1965) sphere results in a radial profile that agrees exquisitely well with that of the $f_{M}=0.1$ disc.

\subsection{Enhanced tidal stripping}

By using the same merger tree realizations for all of the halo-disc configurations, SatGen enables us to directly assess the impact of the disc on individual subhaloes. In Fig. 3, we use a log-density heatmap to show the distribution of changes in subhalo mass relative to the no-disc (nd) configuration, expressed via the ratio $m_{\mathrm{d}} / m_{\mathrm{nd}}$, as a function of the most recent orbital pericentric radius, $r_{\mathrm{p}}$. Each panel corresponds to a different final disc mass, as indicated. We also plot the median $m_{\mathrm{d}} / m_{\text {nd }}$ in each $r_{\mathrm{p}}$ bin in order to better highlight the trend. 

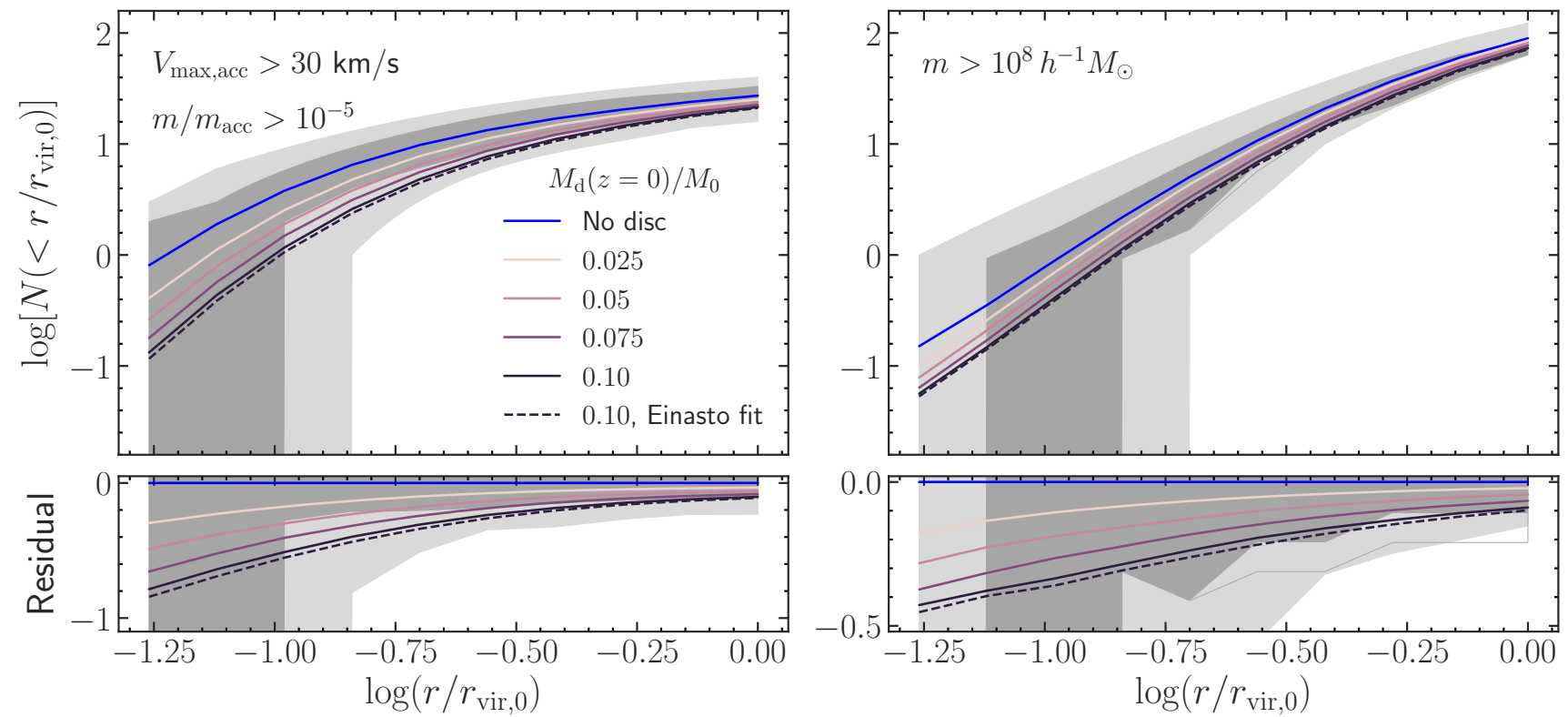

Figure 2. The cumulative radial subhalo abundance profile, which includes subhaloes of all orders with (left-hand panel) $V_{\max }$ acc $>30 \mathrm{~km} \mathrm{~s}{ }^{-1}$ and $m / m_{\text {acc }}>$ $10^{-5}$ or (right-hand panel) $m>10^{8} h^{-1} \mathrm{M}_{\odot}$ at $z=0$. The meanings of the curves and shaded regions are consistent with those of Fig. 1. The disc dramatically reduces the mean abundance of galaxy-hosting subhalo candidates in the halo interior. None the less, the mean curves of all disc configurations lie within the halo-to-halo variance of the disc-less results.
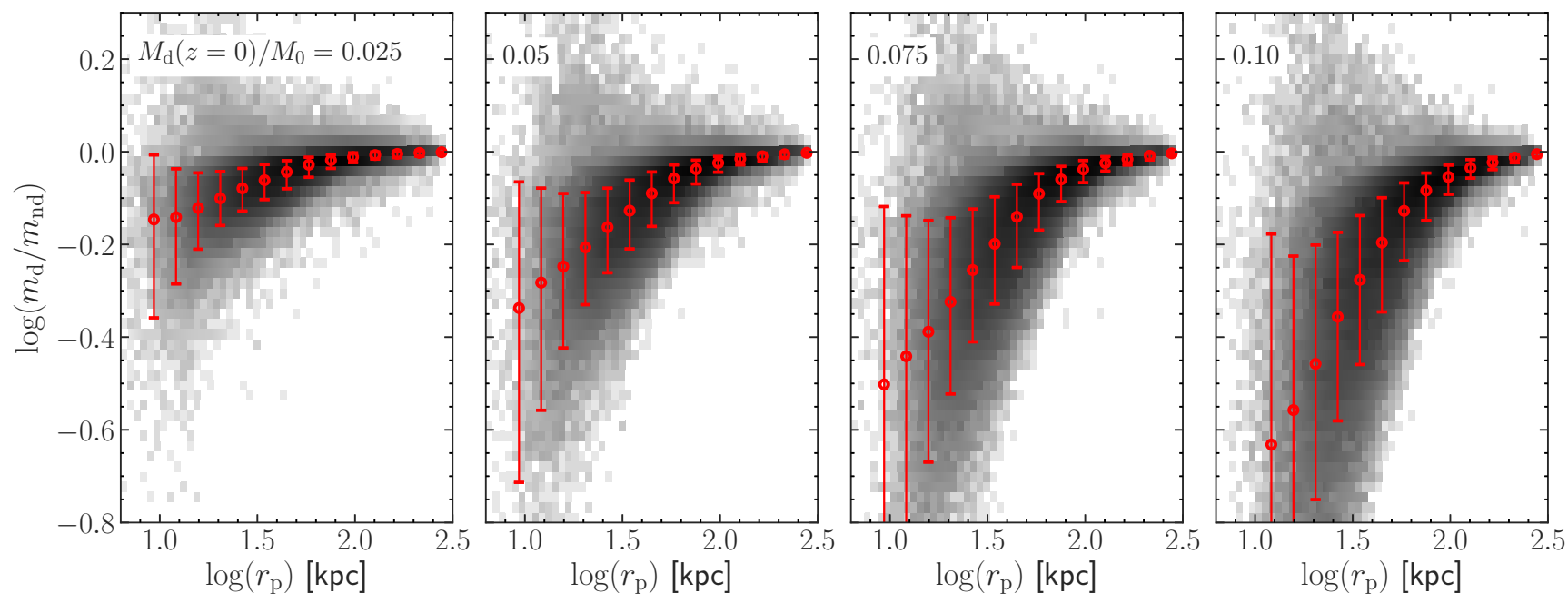

Figure 3. The pericentric radius-dependent impact of a disc on the $z=0$ mass of individual subhaloes. Each panel denotes a different final disc mass, with the remaining disc parameters held to their fiducial values. The most recent pericentric radius, $r_{\mathrm{p}}$, is measured directly from the SatGen outputs of the halo-only configuration. First-order subhaloes with $V_{\max , \text { acc }}>30 \mathrm{~km} \mathrm{~s}^{-1}, r<r_{\mathrm{vir}, 0}$, and $m / m_{\mathrm{acc}}>10^{-5}$ in both the halo-only and halo-disc configuration that have experienced at least one pericentric passage are included. Here, $m_{\mathrm{d}} / m_{\mathrm{nd}}$ denotes the ratio between the mass of a particular subhalo in the halo-disc and no-disc configuration. The density heatmap colour is presented on a logarithmic scale. The red circles denote the median $m_{\mathrm{d}} / m_{\mathrm{nd}}$ in each $r_{\mathrm{p}}$ bin, whereas the error bars correspond to the 68 per cent interval of halo-to-halo variance. The disc enhances the mass-loss of subhaloes that pass near the halo centre while having little impact on those with large- $r_{\mathrm{p}}$ orbits.

The $r_{\mathrm{p}}$ are measured directly from the subhalo position data stored in the SatGen snapshots. For the purpose of this plot, we measure the $r_{\mathrm{p}}$ of each subhalo from the disc-less configuration. However, we acknowledge that the $r_{\mathrm{p}}$ of each subhalo is slightly reduced in the presence of a disc, ${ }^{3}$ an effect which itself drives a minor enhancement

${ }^{3}$ For example, the median (top 1 per cent) reduction in $r_{\mathrm{p}}$ due to the $f_{M}=0.1$ disc is $0.004 \mathrm{dex}(0.045 \mathrm{dex})$. in mass-loss. The population of subhaloes included in this analysis have $V_{\max , \text { acc }}>30 \mathrm{~km} \mathrm{~s}^{-1}$, are first-order at $z=0$, have $r<r_{\text {vir, } 0}$ and $m / m_{\text {acc }}>10^{-5}$ at $z=0$ in both the halo-only and halo-disc configuration, and must have experienced at least one pericentric passage. The figure clearly demonstrates that subhaloes that pass closer to the halo centre experience greater mass-loss due to the enhanced central density of the halo-disc system than those that are confined to the halo outskirts. While the median $m_{\mathrm{d}} / m_{\text {nd }}$ begins to 

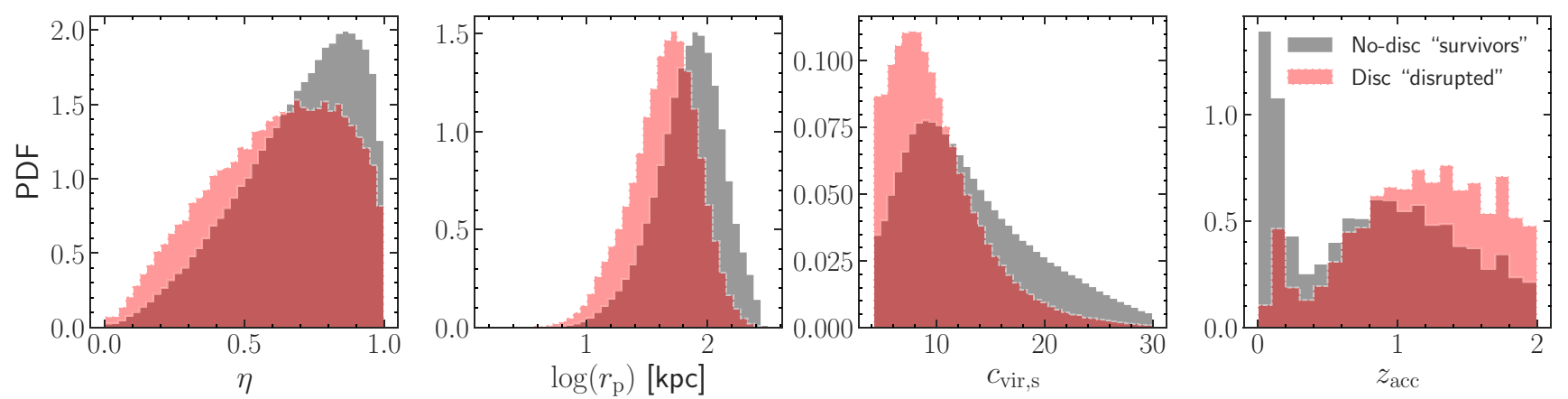

Figure 4. From left to right, the normalized distributions of the instantaneous orbital circularity, $\eta$, radius of first pericentric passage, $r_{\mathrm{p}}$, subhalo concentration at accretion, $c_{\mathrm{vir}, \mathrm{s}}$, and redshift of accretion, $z_{\mathrm{acc}}$, for two groups of subhaloes in the no-disc configuration. The 'survivors' group consists of all first-order subhaloes with $m / M_{0}>10^{-4}$ and $r<r_{\mathrm{vir}, 0}$ at $z=0$ in the no-disc configuration. The 'disrupted' group is the subset of the no-disc 'survivors' that have 'disrupted' (i.e. $m / M_{0}<10^{-4}$ at $z=0$ ) in the composite halo-disc configuration with $f_{M}=0.1$. The subhaloes that do not survive the additional disc-driven mass-loss tend to be on more radial orbits that penetrate more closely into the host centre (smaller mean $\eta$ and $r_{\mathrm{p}}$ ), are less centrally concentrated, and are typically accreted earlier than the no-disc 'survivors'.

deviate from unity for $r_{\mathrm{p}} \lesssim 50-75 \mathrm{kpc}$ in all cases, there is a minor indication that the influence of the disc extends out to further radii as its mass is increased. At $r_{\mathrm{p}} \approx 15 \mathrm{kpc}$, a disc with $f_{M}=0.025(0.1)$ drives an additional $\sim 25$ per cent (70 per cent) loss of subhalo mass on the median. These results are consistent with a similar analysis by Jiang et al. (2021), although we emphasize that we have used a rather different subhalo selection function in this work. Note that the introduction of the disc slightly changes the orbital period, and hence the orbital phase at $z=0$, of all subhaloes - this effect results in a small fraction of cases where the subhalo mass is actually larger than its counterpart in the disc-less realization at $z=0$.

In simulation-based subhalo studies, the particle resolution imposes a fixed lower mass limit on the subhalo population. An unfortunate limitation of this approach is that a subhalo is typically inferred to have been 'disrupted' after its mass falls below this limit. We have argued in previous studies (van den Bosch \& Ogiya 2018; Green et al. 2021) that much of this disruption is not physical and is instead a consequence of the simulation mass limit and artificially enhanced by runaway numerical instabilities. Hence, it is instructive to impose a fixed mass limit on the SatGen results in order to study the properties of subhaloes whose status as 'disrupted' can be specifically traced to the presence of a disc. Here, we define the set of no-disc 'survivors' to be all first-order subhaloes with $m / M_{0}>$ $10^{-4}$ and $r<r_{\mathrm{vir}, 0}$ at $z=0$ in the no-disc realization. The 'disrupted' group is the subset of the 'survivors' that instead have $m / M_{0}<10^{-4}$ at $z=0$ in the halo-disc realization with $f_{M}=0.1$ (i.e. the disruption of these subhaloes can be attributed to the presence of the disc). In Fig. 4, we present the normalized distributions of several orbital and accretion properties of subhaloes in these two groups. Subhaloes that are most vulnerable to additional disc-driven mass-loss, and hence would be preferentially 'disrupted' by the disc in a simulation, are simply those on more radial orbits (i.e. smaller circularity, $\eta$, as defined in Wetzel 2011) that pass closer to the halo centre (smaller $r_{\mathrm{p}}$ ), are less centrally concentrated at accretion (smaller $c_{\mathrm{vir}, \mathrm{s}}$ ), and have undergone tidal evolution for a longer period of time (larger $\left.z_{\text {acc }}\right)$. We expand on the implications of these relatively intuitive findings in the discussion of 'disc shocking' (Section 4). Note that a consequence of the preferential 'disruption' of radially orbiting subhaloes due to the introduction of the disc is a minor change in the subhalo velocity anisotropy towards more circular orbits. The velocity anisotropy profiles predicted by SatGen will be the focus of an upcoming follow-up study.

\subsection{Azimuthal bias of subhaloes}

It is well known that satellite galaxies are preferentially distributed along the major axis of their central host galaxy (e.g. Brainerd 2005; Yang et al. 2006; Azzaro et al. 2007). This 'azimuthal bias' is typically interpreted as implying that central galaxies are aligned with their host haloes. In particular, numerous studies have pointed out that subhaloes in DM simulations are preferentially distributed along the major axis of their host halo (e.g. Knebe et al. 2004; Libeskind et al. 2005; Zentner et al. 2005b). Although a small part of this alignment can be attributed to the preferred direction of subhalo accretion along large-scale filaments (e.g. Aubert, Pichon \& Colombi 2004; Faltenbacher et al. 2008; Morinaga \& Ishiyama 2020), it is mainly due to the fact that host haloes themselves are not spherical (e.g. Wang et al. 2005; Agustsson \& Brainerd 2006; Wang et al. 2008). Hence, as long as central galaxies are roughly aligned with their host haloes, this non-spherical distribution of subhaloes naturally explains the azimuthal bias in the observed distribution of satellite galaxies (e.g. Agustsson \& Brainerd 2006; Kang et al. 2007).

However, since the central galaxy influences the tidal evolution of the subhaloes, an alternative explanation for the azimuthal bias may be that the central (disc) galaxy preferentially destroys satellite galaxies along more polar-inclined orbits. This would introduce deviations from azimuthal symmetry even when the host halo is spherical and subhaloes are accreted isotropically (as in SatGen).

In order to explore this, we search for an angular bias in our predicted subhalo population as a function of disc mass. In SatGen, the cylindrical $z$-axis is the disc axis of symmetry; hence, a discdriven bias should manifest itself in the form of an asymmetry in the number of polar subhaloes, with $|\cos (\theta)|>0.5$, and planar subhaloes, with $|\cos (\theta)|<0.5$, where $\theta$ is the host-centric spherical polar angle. We restrict our sample to first-order subhaloes with $m / M_{0}>10^{-4}$ at $z=0$ that lie within $50 \mathrm{kpc}$ of the host centre, but we emphasize that we considered a range of subhalo selection functions and bounding radii and found qualitatively identical results. In order to compute a robust estimate of the mean polar subhalo fraction, $\langle N(|\cos (\theta)|$ $>0.5) / N\rangle$, and its uncertainty, we stack our subhalo sample over the ensemble of hosts and perform bootstrap resampling. For each disc mass, we generate 2000 bootstrap estimates of $N(|\cos (\theta)|>$ $0.5) / N$ and present the 2.5-97.5 percentile intervals in Fig. 5. Note the complete lack of any significant azimuthal bias; on average, there are equal numbers of 'polar' and 'planar' subhaloes. 


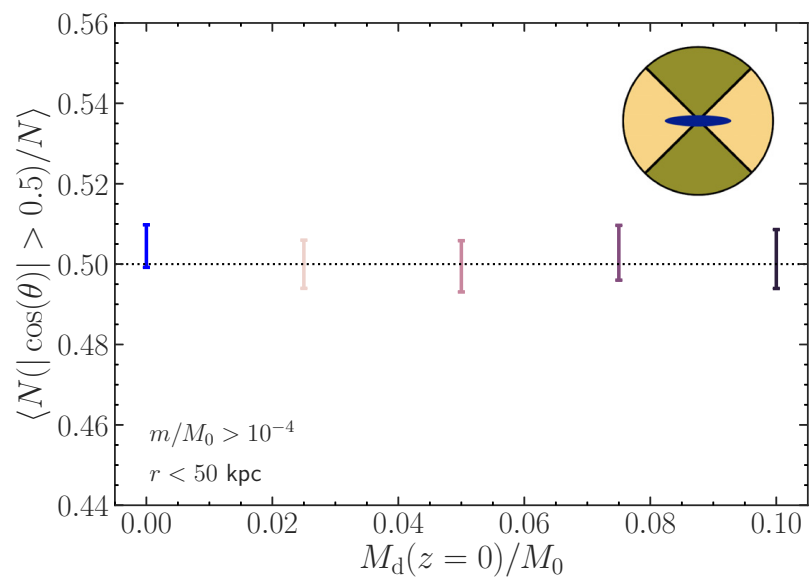

Figure 5. The polar subhalo fraction as a function of the disc mass fraction. The sample includes first-order subhaloes with $m / M_{0}>10^{-4}$ at $z=0$ that lie within $50 \mathrm{kpc}$ of the host centre and is stacked over the ensemble of hosts. The estimated uncertainty range of the fraction of polar subhaloes, which have host-centric polar angles that satisfy $|\cos (\theta)|>0.5$ (i.e. those in the green region of the schematic in the upper right), is computed via bootstrap resampling. The error bars denote the $2.5-97.5$ percentile intervals of the 2000 bootstrap estimates of the polar fraction. We find no statistically significant disc-driven azimuthal bias in subhalo positions, regardless of disc mass.

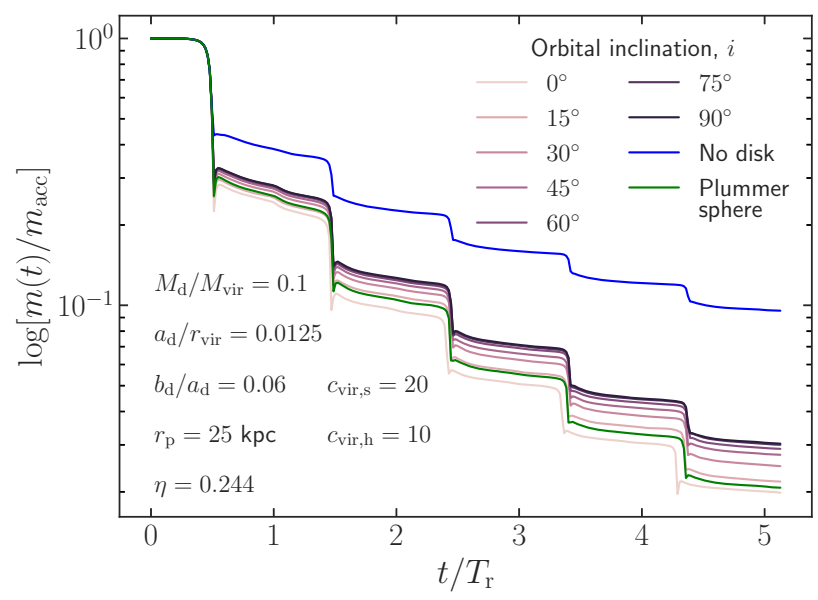

Figure 6. The $m(t) / m_{\text {acc }}$ trajectories of subhaloes in idealized $N$-body simulations (see Appendix A). The times are normalized by the radial orbital period, $T_{r}$. The disc mass, disc shape, host and subhalo concentrations, orbital energy, and orbital angular momentum are all held fixed as we vary the orbital inclination angle from $0^{\circ}$ (the orbit is in the plane of the disc) to $90^{\circ}$ (perpendicular to the plane of the disc). As the orbit becomes less inclined, the cumulative mass-loss increases, but the overall inclination dependence is weak. Replacing the disc potential with a Plummer (1911) sphere (with the same total mass and a nearly equivalent spherically enclosed mass profile) yields greater mass-loss than those of the inclined orbits in the presence of a disc, demonstrating the insignificance of 'disc shocking'.

We ascribe this lack of azimuthal bias to two effects. First of all, the mass-loss of subhaloes depends only weakly on the (polar) angle of incidence between the orientation of the disc and the subhalo orbit. This is demonstrated explicitly in Fig. 6, which shows the $m(t) / m_{\text {acc }}$ trajectories of subhaloes in idealized $N$-body simulations (see Appendix A for details). Different curves correspond to different orbital inclinations, $i=90^{\circ}-\theta$, of the initial orbital plane, as indicated, with all other parameters kept fixed. Note that the chosen orbit is highly eccentric, with a small pericentric radius of $r_{\mathrm{p}}=25 \mathrm{kpc}$ and orbital circularity of $\eta=0.244$. For comparison, the blue curve shows the corresponding result in the absence of a central disc and the green curve corresponds to the case where the disc has been replaced by a Plummer (1911) sphere with a nearly equivalent spherically enclosed mass profile, which we discuss in Section $4 .{ }^{4}$ Note that more planar orbits (i.e. those with smaller $i$ ) result in slightly more mass-loss. Hence, if anything, disc-driven disruption should result in a deficit of planar satellites relative to polar satellites, opposite to the trend seen in observational data. The fact that no azimuthal bias emerges is owed to the fact that in an axisymmetric potential the subhalo is not confined to an orbital plane; unlike an orbit in a spherical potential, its polar angle evolves with time. This washes out the weak dependence on the (initial) inclination seen in Fig. 6.

To summarize, we conclude that the angular bias observed in the azimuthal distribution of satellite galaxies does not have its origin in a disc-driven preferential disruption of subhaloes along more polarinclined orbits. Rather, it is simply due to the central galaxy being aligned with the moment of inertia of the non-spherical host halo combined with the existence of a preferred direction of subhalo accretion due to large-scale filaments.

\subsection{Dependence on disc parameters}

In all previous results, we have studied disc configurations with various final masses (controlled by $f_{M}$ ) but with only the fiducial $f_{a}$, $\beta_{a}, \beta_{M}$, and $b_{\mathrm{d}} / a_{\mathrm{d}}$. In Fig. 7, we use a summary statistic to demonstrate that our results are insensitive to these other parameters, which control the disc growth and size. For each disc configuration, we compute the mean number of subhaloes (with $V_{\max }$ acc $>30 \mathrm{~km} \mathrm{~s}^{-1}$ and $m / m_{\text {acc }}$ $>10^{-5}$ ) enclosed within $50 \mathrm{kpc}$ of the halo centre at $z=0$ (averaged over all 10000 trees), which we denote $\langle N(r<50 \mathrm{kpc})\rangle$. In panels 1 , $2,3,5$, and 6 , we vary one of the disc parameters, fixing the other four to a set of baseline values $\left(f_{a}=0.0125, \beta_{a}=1 / 3, f_{M}=0.1, \beta_{M}=1\right.$, and $b_{\mathrm{d}} / a_{\mathrm{d}}=0.08$ ). Note that we use the large $f_{M}=0.1$ for our baseline in order to enhance the sensitivity of our results to the other disc parameters. We explore the impact of adiabatic contraction of the host halo in the remaining three panels, which we discuss in Section 3.6. For comparison, the horizontal lines indicate the $\langle N(r<50 \mathrm{kpc})\rangle$ of the no-disc configuration, while the grey shaded regions mark the corresponding 68 per cent and 95 per cent halo-to-halo variance intervals. Once again, the mean result lies within the disc-less halo-tohalo variance for every disc configuration studied. Clearly, the mean subhalo abundance within $50 \mathrm{kpc}$ is minimally impacted by the disc scale height and the rate at which the disc grows (both in physical size and mass) relative to the host halo. It is only slightly sensitive to the disc scale length; a more compact disc suppresses more subhaloes. However, the disc mass is the only parameter that has a strong effect on $\langle N(r<50 \mathrm{kpc})\rangle$ - the mean abundance drops by 23 percent (56 per cent) relative to the disc-less case when $f_{M}=0.025(0.1)$. For comparison, the replacement of the $f_{M}=0.1$ disc with an Einasto (1965) sphere drives a 59 percent suppression in $\langle N(r<50 \mathrm{kpc})\rangle$ relative to the disc-less case. This finding is in excellent agreement

${ }^{4}$ The Plummer scale length that yields the best match to the spherically enclosed mass profile of the MN disc (when $b_{\mathrm{d}} / a_{\mathrm{d}}=0.06$ ) is $\epsilon \approx 0.92 a_{\mathrm{d}}$. The total mass of the two systems is identical but the Plummer (1911) sphere is slightly more centrally concentrated - its enclosed mass is $\sim 10$ per cent larger than that of the disc at $r \approx 10 a_{\mathrm{d}}$. 

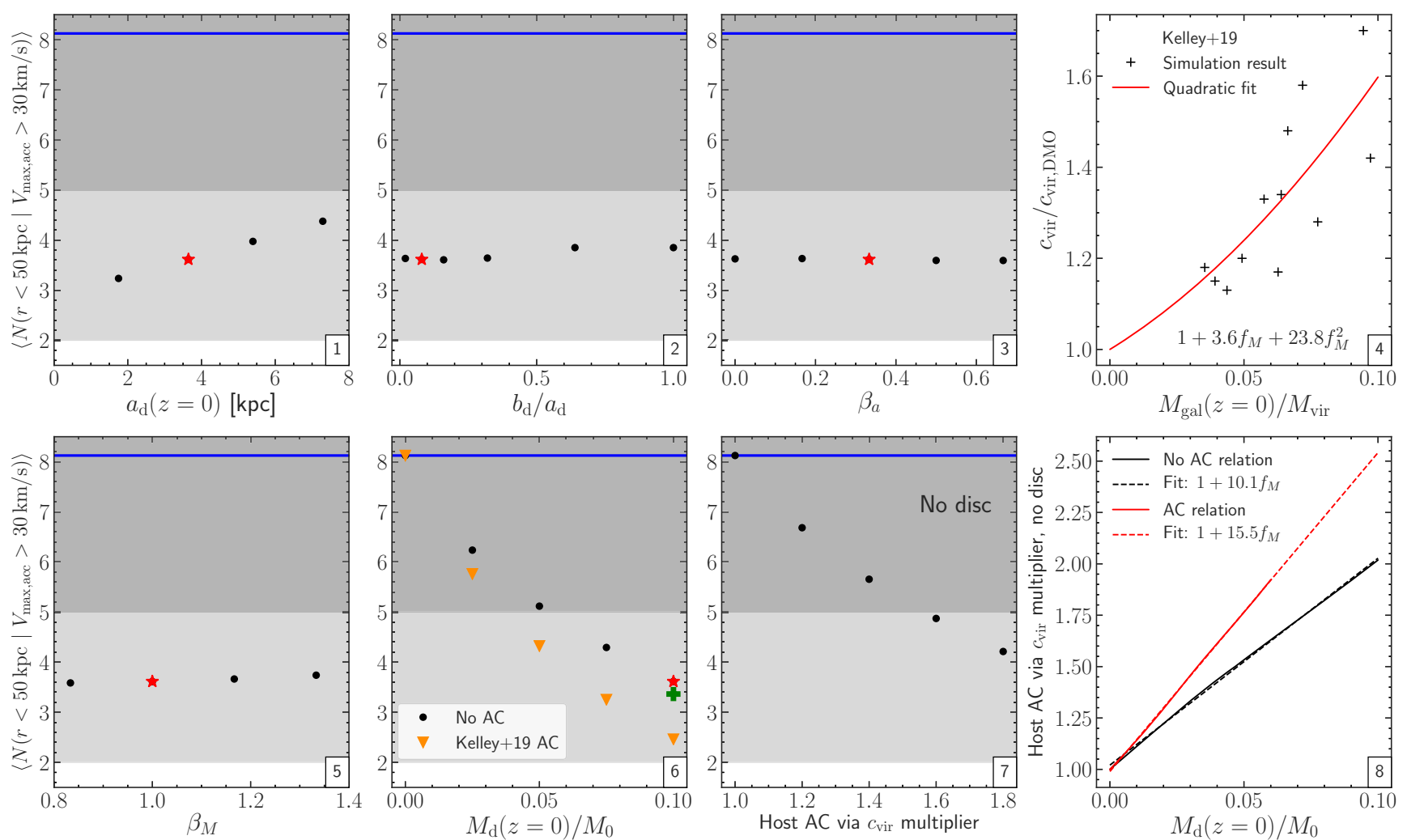

Figure 7. The mean abundance of subhaloes with $V_{\max }$ acc $>30 \mathrm{~km} \mathrm{~s}^{-1}$ and $m / m_{\text {acc }}>10^{-5}$ enclosed within $50 \mathrm{kpc}$ of the halo centre at $z=0$, averaged over all 10000 merger trees. In panels $1,2,3,5$, and 6 , we vary one disc parameter, which includes (i) the disc scale length, $a_{\mathrm{d}}\left(z=0\right.$ ), which is set by $f_{a}$, (ii) the disc scale height, which is expressed as a fraction of the scale length, $b_{\mathrm{d}} / a_{\mathrm{d}}$, (iii) the power-law slope of $a_{\mathrm{d}}(z), \beta_{a}$, (iv) the power-law slope of $M_{\mathrm{d}}(z), \beta_{M}$, and (v) the disc mass fraction, $M_{\mathrm{d}}(z=0) / M_{0}$, which is set by $f_{M}$. The red star corresponds to the baseline disc configuration $\left(f_{a}=0.0125, \beta_{a}=1 / 3, f_{M}=0.10, \beta_{M}=1\right.$, and $\left.b_{\mathrm{d}} / a_{\mathrm{d}}=0.08\right)$ around which the parameters are varied. The green cross indicates the spherical Einasto (1965) substitute for the baseline disc. The blue line denotes the $\langle N(r<50 \mathrm{kpc})\rangle$ of the halo-only configuration, which is surrounded by its 68 per cent and 95 per cent halo-to-halo variance intervals (dark and light shaded regions, respectively). Consistent with Garrison-Kimmel et al. (2017), only the disc mass has a strong impact on the subhalo statistics. Panel 4 displays the relationship (and corresponding quadratic fit) between the mass fraction of a central galactic potential and the corresponding boost in the NFW concentration of the host due to adiabatic contraction seen in the cosmological simulations of Kelley et al. (2019). This host contraction due to the disc is accounted for in the orange triangles in panel 6. The impact of an increased host concentration in the absence of a disc is shown in panel 7. The degeneracy between host contraction (without a disc) and a disc potential (both with and without adiabatic contraction) is demonstrated in panel 8 - increasing the disc mass fraction by 0.01 and ignoring (accounting for) adiabatic contraction due to the disc has the same effect on $\langle N(r<50 \mathrm{kpc})\rangle$ as that of a 10 per cent (15.5 per cent) increase in the host concentration.

with the cosmological simulation study of Garrison-Kimmel et al. (2017), who embed a variety of different disc potentials into their host haloes, finding that only the total mass of the disc has a significant impact on the resulting subhalo statistics.

\subsection{Adiabatic contraction of the host}

Thus far, we have neglected to consider the adiabatic contraction of the host halo due to the formation of the galactic disc. This simplification has enabled us to assess the relative impact of a disc potential on the subhalo population while keeping all other properties of the host consistent with its disc-less counterpart. In addition, proper modelling of adiabatic contraction due to the growth of an axisymmetric potential itself remains an open problem. For example, the 'standard' adiabatic invariant, $r M(r)$, originally suggested by Barnes \& White (1984) and Blumenthal et al. (1986) and often used in modelling disc galaxies and their rotation curves (e.g. Mo, Mao \& White 1998; van den Bosch \& Swaters 2001), is only valid for unrealistic, completely spherical systems in which all particles move on circular orbits. Furthermore, the fact that the scatter in the TullyFisher relation is independent of size (e.g. Courteau \& Rix 1999; Courteau et al. 2007) has been used to argue that disc formation cannot be associated with significant halo contraction (e.g. Dutton et al. 2007), which might have its origin in non-adiabatic processes operating during disc formation (see e.g. El-Zant, Shlosman \& Hoffman 2001; Tonini, Lapi \& Salucci 2006).

Despite these issues, we now proceed to investigate how adding (adiabatic) contraction of the host halo due to the assembly of the disc impacts the subhalo population. Rather than assuming a particular adiabatic invariant, we consider a simplified model of the halo contraction based on the simulation results of Kelley et al. (2019). These authors run a suite of twelve dark matter-only cosmological zoom-in simulations, each of which is centred on a different Milky Way-like halo. They then re-run each of the simulations with an embedded galactic potential, which grows over time, placed at the centre of each halo. They fit an NFW profile to each host halo at $z=$ 0 in both the halo-only and halo-disc configurations and report the concentrations ( $c_{\mathrm{vir}, \text { DMO }}$ and $c_{\mathrm{vir}}$, respectively). In panel 4 of Fig. 7 , 
we plot the ratio, $c_{\mathrm{vir}} / c_{\mathrm{vir}, \text { DMO }}$, as a function of the fraction of mass in the embedded potential, $M_{\mathrm{gal}}(z=0) / M_{\mathrm{vir}}$. Note that Kelley et al. (2019) model the galaxy as a composite potential that consists of a stellar disc, a gaseous disc, and a stellar bulge; we define $M_{\text {gal }}$ to be the combined mass of these three systems and compare it directly with the mass of our single-component stellar disc. Clearly, when the central galaxy makes up a larger fraction of the total host mass, the host experiences greater contraction, which corresponds to a larger

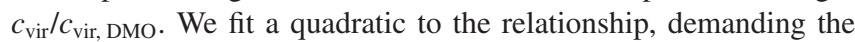
physical constraint that $c_{\mathrm{vir}} / c_{\mathrm{vir}}$ DMO $=1$ when $M_{\mathrm{gal}}(z=0) / M_{\mathrm{vir}}=0$. We use this fit as the basis of our adiabatic contraction model.

We emulate adiabatic contraction using the following approach. Given the $f_{M}$ of the disc potential of interest, we look up the corre-

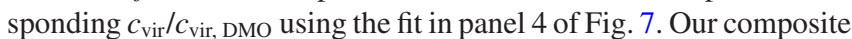
host system is exactly the same as before except that the concentration of the NFW host halo is multiplied by the corresponding value of $c_{\mathrm{vir}} / c_{\mathrm{vir}}$ DMO at all times during the evolution of the subhaloes. For $f_{M}=0.05(0.1)$, the host concentration is boosted by a factor of 1.24 (1.60). Note that the total mass of the composite system enclosed within $r_{\text {vir }}$ remains unchanged. In panel 6 of Fig. 7, the orange triangles show the mean subhalo abundance within $50 \mathrm{kpc}$ of the host centre, $\langle N(r<50 \mathrm{kpc})\rangle$ (a good summary of the overall influence of the disc), as a function of $f_{M}$ when the host concentration is boosted in order to account for adiabatic contraction. These can be directly compared to the case without adiabatic contraction for each $f_{M}$, denoted by the black circles. As expected, as the disc becomes more massive, the relative impact of the host contraction becomes more significant. Relative to the halo-only host, the $f_{M}=0.05(0.1)$ disc suppresses $\langle N(r<50 \mathrm{kpc})\rangle$ by 37 per cent $(56$ per cent) without adiabatic contraction and by 47 per cent ( 70 per cent) when the host concentrations are boosted according to the Kelley et al. (2019) model. Note that the $\langle N(r<50 \mathrm{kpc})\rangle$ of the most massive disc remains within the halo-to-halo variance of the halo-only configuration even when adiabatic contraction is taken into account, further demonstrating the importance of such variance in subhalo statistics.

In Section 2, we claimed that the impact of adiabatic contraction is degenerate with simply increasing the disc mass. The reason for this is simple: increasing the disc mass increases the central concentration of the host mass, which is the same result as that of adiabatic contraction. In panel 7 of Fig. 7, we consider how $\langle N(r<50 \mathrm{kpc})\rangle$ of the halo-only configuration is suppressed if we boost the host concentration by a constant factor at all times during subhalo evolution. In the absence of a disc, boosting the host concentration by a factor of 1.6 drives a 40 per cent reduction in $\langle N(r<50 \mathrm{kpc})\rangle$. This level of adiabatic contraction is seen in systems with a disc with $f_{M}=0.1$, which itself suppresses $\langle N(r<50 \mathrm{kpc})\rangle$ by 56 percent without the concentration boost. Hence, the effect of the disc itself and the adiabatic contraction that it brings about are both of similar importance, although the disc drives slightly more subhalo suppression. Since the host contraction and disc mass are degenerate, we can use both of panels 6 and 7 in Fig. 7 in order to understand the relationship between disc-driven suppression (both with and without also accounting for adiabatic contraction; in terms of $\langle N(r<50 \mathrm{kpc})\rangle)$ and concentration boostdriven suppression (in the absence of a disc). We interpolate between both the black circles and orange triangles in panel 6 and the black circles in panel 7 and then perform the following: for each value of $M_{\mathrm{d}}(z=0) / M_{0}$ (separately with and without adiabatic contraction), we find the value of the host $c_{\text {vir }}$ multiplier (without a disc) that corresponds to the same $\langle N(r<50 \mathrm{kpc})\rangle$. These two relationships are shown in panel 8 of Fig. 7 alongside nearly perfect linear fits. In terms of $\langle N(r<50 \mathrm{kpc})\rangle$, increasing the disc mass fraction by 0.01 and ignoring (accounting for) adiabatic contraction due to the disc has the same effect as a 10 per cent ( 15.5 per cent) increase in the host concentration. Hence, the impact of a disc potential can be roughly emulated by simply making the host halo more concentrated.

\section{DISCUSSION}

As we have seen, the presence of a central disc galaxy causes a suppression in the abundance of subhaloes. Our SatGen-based results are in good agreement with previous results based on $\mathrm{N}$-body simulations (D'Onghia et al. 2010; Errani et al. 2017; GarrisonKimmel et al. 2017; Sawala et al. 2017; Kelley et al. 2019), both qualitatively and quantitatively. However, we disagree with D'Onghia et al. (2010) and Garrison-Kimmel et al. (2017) with regards to the importance and origin of this disc-induced substructure depletion. In particular, contrary to these previous studies, we argue that the presence of a disc does not cause actual, physical disruption of substructure. Rather, it merely causes enhanced stripping. We emphasize that this is not just a semantic issue; rather, it is the difference between having no substructure within the inner $30 \mathrm{kpc}$ of the Milky Way and having thousands of low mass subhaloes (a small fraction of which may host a satellite galaxy) with reduced mass compared to a case without a disc.

In particular, D'Onghia et al. (2010) argued that subhaloes that pass near a central disc galaxy will be destroyed due to impulsive 'disc shocking'. Whenever a subhalo passes through the plane of a disc at sufficiently high speed, its internal energy will increase by an amount $\Delta E$ that can be calculated analytically (Ostriker, Spitzer \& Chevalier 1972; Binney \& Tremaine 2008). The crux of the argument made by D'Onghia et al. (2010) is that whenever this $\Delta E$ exceeds the binding energy of the subhalo, $\left|E_{\mathrm{b}}\right|$, the subhalo is 'certain' to be disrupted. They proceed to show that this condition is met by a significant fraction $(\sim 15$ per cent) of subhaloes in a realistic, MWlike system.

However, as shown in van den Bosch et al. (2018), it is incorrect to assume that $\Delta E>\left|E_{\mathrm{b}}\right|$ will result in disruption. What matters is not only the total energy injected but also how that energy is distributed over the constituent particles. Since $\Delta E \propto l^{2}$, the particles in the outskirts of the subhalo, which need little energy to escape, receive the bulk of the energy injection whereas the particles near the subhalo centre, which need a large amount of energy to escape, receive virtually no energy. As a consequence, subhaloes can actually experience a tidal shock that exceeds many multiples of their binding energy and still survive. In fact, van den Bosch et al. (2018) show that subhaloes with an NFW profile that experience a tidal shock with $\Delta E /\left|E_{\mathrm{b}}\right|=1$ (10) only lose roughly 20 per cent (55 per cent) of their mass.

Another argument against disc shocking is the fact that in most cases it is subdominant to 'halo shocking', which is tidal heating due to a high speed pericentric passage with respect to the host halo itself. Indeed, van den Bosch et al. (2018) showed that the average $\Delta E /\left|E_{\mathrm{b}}\right|$ of a subhalo due to its first pericentric passage in a (disc-less) host halo is about $\sim 1.9$, which is larger than the average $\Delta E /\left|E_{\mathrm{b}}\right|$ due to disc shocking. This is consistent with D'Onghia et al. (2010), who showed that halo shocking dominates over disc shocking in a typical MW-like system except for subhaloes with pericentric radii smaller than $\sim 10 \mathrm{kpc}$.

The insignificance of disc shocking is also evident from Fig. 6. If disc shocking were indeed the dominant factor in disc-driven subhalo depletion, then a subhalo on a highly inclined orbit (relative to the disc) should experience more mass-loss than a subhalo whose orbit is confined to the plane of the disc. However, Fig. 6 demonstrates that 
the exact opposite trend is seen in our idealized $N$-body simulations (see Appendix A), which is nicely reproduced by our tidal stripping model that is implemented in SatGen. Furthermore, replacing the disc potential with an equivalent Plummer (1911) sphere in an idealized simulation (see Fig. 6) results in greater mass-loss than those of the inclined orbits in the presence of a disc. This is consistent with our SatGen results, where Figs 1, 2, and 7 demonstrate that subhalo statistics are generally insensitive to the replacement of the disc with a spherical system that has an equivalent spherically enclosed mass profile.

In fact, our tidal stripping-based mass-loss model successfully reproduces the subhalo mass evolution of the idealized halo-only DASH simulations, our new composite halo-disc simulations (Appendix A), and a wide range of results from the Bolshoi cosmological simulations (Green et al. 2021) without an explicit prescription for tidal shocking. The model relies upon the tight empirical relationship between the stripped subhalo density profile and the bound mass fraction, which is independent of the details of how the mass was lost (e.g. Hayashi et al. 2003; Peñarrubia et al. 2008; Green \& van den Bosch 2019). The accuracy of our mass-loss model and the strength of the density profile-mass fraction relationship would be substantially reduced if tidal shocking dominated for some orbits (i.e. small $r_{\mathrm{p}}$ ) and tidal stripping dominated for others (i.e. large $r_{\mathrm{p}}$ ).

Based on all of these considerations, we conclude that disc shocking plays, at most, a minor role in the substructure suppression caused by a disc potential. Instead, the presence of a disc greatly increases the central mass concentration of the host, which results in an overall increase in tidal stripping that becomes increasingly significant as $r_{\mathrm{p}}$ becomes smaller. This net increase in subhalo massloss effectively shifts the mean SHMF to the left (see Fig. 1). The total subhalo abundance above a particular simulation mass limit is decreased. However, considering the fact that a NFW subhalo should never fully disrupt (see e.g. van den Bosch et al. 2018; Errani \& Peñarrubia 2020; Errani \& Navarro 2021), we emphasize that this reduced abundance is not due to 'disruption', but is instead a consequence of enhanced mass-loss combined with a fixed resolution limit.

\section{SUMMARY}

The demographics of DM substructure depend on both the particle nature of DM and the gravitational interaction between DM and baryons. Hence, in order to understand the dependence on the former, we must be able to properly account for the latter. Much progress has been made towards correctly capturing the manner in which baryons shape the overall DM distribution (e.g. D'Onghia et al. 2010; Zolotov et al. 2012; Brooks et al. 2013; Garrison-Kimmel et al. 2017; Sawala et al. 2017; Kelley et al. 2019). However, a common limitation of these studies is that they are all based on expensive cosmological simulations, which has limited their ability to consider statistically complete halo samples and properly contextualize results in terms of the corresponding halo-to-halo variance. The primary finding of these works is clear: the presence of a galactic disc suppresses subhalo abundance, an effect that becomes stronger towards the halo centre. Since the suppression also increases with increasing disc mass, properly accounting for this disc-driven subhalo depletion is especially important for Milky Way-mass systems, which sit at the peak of the stellar mass-halo mass relation.

In this paper, we used the SatGen semi-analytical modelling framework to assess the impact of a galactic disc potential on the DM subhalo demographics of MW-like hosts. This method is not impacted by issues related to numerical disruption, which still hamper the results of $\mathrm{N}$-body simulations, and allows for the construction of large halo samples, which enables unprecedented statistical power. Using an ensemble of 10000 merger trees with $M_{0}=10^{12} h^{-1} \mathrm{M}_{\odot}$, we generated an equally large sample of evolved subhalo populations using a range of different composite halodisc potentials. This approach allowed us to isolate the differential influence of the disc by controlling for assembly history variance. Leveraging the computational efficiency of SatGen, we explored a wide range of disc parameter space, spanning the disc mass, size, and formation history. We used the resulting subhalo catalogues to study subhalo mass functions and radial abundance profiles. We also measured the relative impact of the disc on the $z=0$ mass of individual subhaloes as well as examined whether disc-driven subhalo depletion gives rise to an azimuthal bias in the spatial distribution of the subhalo population. Our most notable findings are summarized as follows:

(i) For a disc mass fraction of $f_{M}=0.05$, which is a typical value for a Milky Way-sized halo, the normalization of the mean SHMF (of subhaloes with $r<r_{\text {vir, } 0}$ ) is suppressed by $\lesssim 10$ per cent relative to the no-disc case. When only considering subhaloes within $50 \mathrm{kpc}$ of the halo centre, the mean SHMF normalization is decreased by $\sim 30$ per cent. The level of substructure suppression increases with disc mass. However, the mean disc-driven impact on the SHMF is dwarfed by the halo-to-halo variance in all cases.

(ii) The disc has a considerably larger influence on the subhalo abundance near the halo centre, as evidenced by the mean radial subhalo abundance profiles. For example, the mean abundance of potential galaxy-hosting subhaloes (with $V_{\max \text {, acc }}>30 \mathrm{~km} \mathrm{~s}^{-1}$ ) is suppressed by $\sim 40$ per cent within $50 \mathrm{kpc}$ of the halo centre relative to the no-disc case when $f_{M}=0.05$ but is reduced by $\sim 60$ per cent within $20 \mathrm{kpc}$. The mean effect of the disc on the radial profile is again eclipsed by the halo-to-halo variance.

(iii) By tracking individual subhaloes across different host halodisc configurations, we have shown that the presence of a central disc causes excess subhalo mass-loss, the strength of which increases with decreasing pericentric radius. For example, at $r_{\mathrm{p}} \approx 50 \mathrm{kpc}(20 \mathrm{kpc})$, a disc with $f_{M}=0.05$ drives an additional $\sim 15$ per cent ( 40 per cent) loss of subhalo mass on the median.

(iv) By imposing a fixed mass resolution limit $\left(\mathrm{m} / \mathrm{M}_{0}>10^{-4}\right)$, consistent with simulation-based subhalo studies, we analysed the orbital and accretion properties of subhaloes that survive until $z=0$ in the absence of a disc but are 'disrupted' (i.e. their $m$ falls below the mass cut) by $z=0$ in the composite host halo-disc case. On average, these disc-disrupted subhaloes are found to have smaller $r_{\mathrm{p}}$ and $c_{\mathrm{vir}, \mathrm{s}}$ than the overall sample.

(v) The presence of the disc does not cause an azimuthal bias in the spatial distribution of subhaloes for any of the disc masses considered $\left(f_{M} \leq 0.1\right)$. Therefore, the observed alignment of satellite galaxies with the orientation of their central host is not driven by the presence of a disc, but is instead an outcome of galaxy-halo alignment in non-spherical haloes.

(vi) The overall amplitude of disc-driven subhalo depletion is relatively insensitive to the size of the disc and its detailed formation history (both in terms of its size and mass). Rather, it depends almost exclusively on the final mass of the disc. The replacement of the disc with a spherical system, which has a nearly equivalent spherically enclosed mass profile, of the same total mass yields subhalo statistics that are in excellent agreement with the analogous halo-disc configuration.

(vii) We demonstrated that the impact of a disc potential can be emulated by simply increasing the concentration of the host halo. 
Increasing the disc mass fraction by 0.01 and ignoring (accounting for) adiabatic contraction due to the disc has the same impact on $\langle N(r<50 \mathrm{kpc})\rangle$ as boosting the host concentration by 10 percent ( 15.5 per cent). Adiabatic contraction of the host due to the formation of the galactic disc only has a significant effect on the overall subhalo abundance when the disc mass fraction is large.

Overall, our SatGen-based results are in excellent agreement (both qualitatively and quantitatively) with previous results based on $N$-body simulations (D'Onghia et al. 2010; Errani et al. 2017; Garrison-Kimmel et al. 2017; Sawala et al. 2017; Kelley et al. 2019). However, as discussed in detail in Section 4, we disagree with the notion promoted by D'Onghia et al. (2010) and Garrison-Kimmel et al. (2017) that the disc causes actual disruption of subhaloes via impulsive disc shocking. Rather, the disc simply increases the density in the central region of the halo, which promotes excess mass-loss. When this enhanced mass-loss results in the subhalo mass dropping below the resolution limit of a numerical simulation, the subhalo appears to have been disrupted; in reality, it would continue to survive with a reduced mass (and should never fully disrupt; see e.g. van den Bosch et al. 2018; Errani \& Peñarrubia 2020; Errani \& Navarro 2021). Another new insight that has emerged from this study relates to the overall importance of disc-driven subhalo depletion. By using a large ensemble of merger trees, we were able to demonstrate that the impact of the disc is small compared to the expected halo-to-halo variance, even for the most massive discs considered. Hence, when using the abundance of satellite galaxies or subhaloes in a single system, such as the Milky Way, it is more important to account for halo-to-halo variance than the impact of the central galaxy when making inferences.

\section{ACKNOWLEDGEMENTS}

The authors thank Uddipan Banik and Raphaël Errani for helpful conversations throughout the development of this work. SBG is supported by the US National Science Foundation Graduate Research Fellowship under Grant No. DGE-1752134. FCvdB is supported by the National Aeronautics and Space Administration through Grant No. 17-ATP17-0028 issued as part of the Astrophysics Theory Program. FJ is supported by the Troesh Fellowship from the California Institute of Technology.

\section{DATA AVAILABILITY}

The updated SatGen library is publicly available on GitHub. ${ }^{5}$

\section{REFERENCES}

Agustsson I., Brainerd T. G., 2006, ApJ, 644, L25

Aubert D., Pichon C., Colombi S., 2004, MNRAS, 352, 376

Azzaro M., Patiri S. G., Prada F., Zentner A. R., 2007, MNRAS, 376, L43

Banik N., Bovy J., Bertone G., Erkal D., de Boer T. J. L., 2021, J. Cosmol. Astropart. Phys., 10, 043

Barnes J., White S. D. M., 1984, MNRAS, 211, 753

Behroozi P., Wechsler R. H., Hearin A. P., Conroy C., 2019, MNRAS, 488, 3143

Binney J., Tremaine S., 2008, Galactic Dynamics, 2nd edn. Princeton Univ. Press, Princeton

Blumenthal G. R., Faber S. M., Flores R., Primack J. R., 1986, ApJ, 301, 27

Bonaca A. et al., 2020, ApJ, 892, L37

${ }^{5}$ https://github.com/shergreen/SatGen/
Bose S. et al., 2017, MNRAS, 464, 4520

Brainerd T. G., 2005, ApJ, 628, L101

Brooks A. M., Kuhlen M., Zolotov A., Hooper D., 2013, ApJ, 765, 22

Bryan G. L., Norman M. L., 1998, ApJ, 495, 80

Burkert A., 2020, ApJ, 904, 161

Chandrasekhar S., 1943, ApJ, 97, 255

Courteau S., Rix H.-W., 1999, ApJ, 513, 561

Courteau S., Dutton A. A., van den Bosch F. C., MacArthur L. A., Dekel A., McIntosh D. H., Dale D. A., 2007, ApJ, 671, 203

D’Onghia E., Springel V., Hernquist L., Keres D., 2010, ApJ, 709, 1138

Dutton A. A., van den Bosch F. C., Dekel A., Courteau S., 2007, ApJ, 654, 27

Einasto J., 1965, Trudy Astrofiz. Inst. Alma-Ata, 5, 87

El-Zant A., Shlosman I., Hoffman Y., 2001, ApJ, 560, 636

Erkal D., Belokurov V., Bovy J., Sanders J. L., 2016, MNRAS, 463, 102

Errani R., Navarro J. F., 2021, MNRAS, 505, 18

Errani R., Peñarrubia J., 2020, MNRAS, 491, 4591

Errani R., Peñarrubia J., Laporte C. F. P., Gómez F. A., 2017, MNRAS, 465, L59

Faltenbacher A., Jing Y. P., Li C., Mao S., Mo H. J., Pasquali A., van den Bosch F. C., 2008, ApJ, 675, 146

Garrison-Kimmel S. et al., 2017, MNRAS, 471, 1709

Giocoli C., Tormen G., Sheth R. K., van den Bosch F. C., 2010, MNRAS, 404, 502

Green S. B., van den Bosch F. C., 2019, MNRAS, 490, 2091

Green S. B., van den Bosch F. C., Jiang F., 2021, MNRAS, 503, 4075

Hayashi E., Navarro J. F., Taylor J. E., Stadel J., Quinn T., 2003, ApJ, 584, 541

Hezaveh Y. D. et al., 2016, ApJ, 823, 37

Jiang F., van den Bosch F. C., 2016, MNRAS, 458, 2848

Jiang F., van den Bosch F. C., 2017, MNRAS, 472, 657

Jiang F., Dekel A., Freundlich J., Romanowsky A. J., Dutton A. A., Macciò A. V., Di Cintio A., 2019, MNRAS, 487, 5272

Jiang F., Dekel A., Freundlich J., van den Bosch F. C., Green S. B., Hopkins P. F., Benson A., Du X., 2021, MNRAS, 502, 621

Kang X., van den Bosch F. C., Yang X., Mao S., Mo H. J., Li C., Jing Y. P., 2007, MNRAS, 378, 1531

Kaplinghat M., Tulin S., Yu H.-B., 2016, Phys. Rev. Lett., 116, 041302

Kelley T., Bullock J. S., Garrison-Kimmel S., Boylan-Kolchin M., Pawlowski M. S., Graus A. S., 2019, MNRAS, 487, 4409

King I., 1962, AJ, 67, 471

Klypin A. A., Trujillo-Gomez S., Primack J., 2011, ApJ, 740, 102

Knebe A., Gill S. P. D., Gibson B. K., Lewis G. F., Ibata R. A., Dopita M. A., 2004, ApJ, 603, 7

Kravtsov A. V., 2013, ApJ, 764, L31

Libeskind N. I., Frenk C. S., Cole S., Helly J. C., Jenkins A., Navarro J. F., Power C., 2005, MNRAS, 363, 146

Li Z.-Z., Zhao D.-H., Jing Y. P., Han J., Dong F.-Y., 2020, ApJ, 905, 177

Lovell M. R., Frenk C. S., Eke V. R., Jenkins A., Gao L., Theuns T., 2014, MNRAS, 439, 300

Miyamoto M., Nagai R., 1975, PASJ, 27, 533

Mo H. J., Mao S., White S. D. M., 1998, MNRAS, 295, 319

More S., van den Bosch F. C., Cacciato M., Skibba R., Mo H. J., Yang X., 2011, MNRAS, 410, 210

Morinaga Y., Ishiyama T., 2020, MNRAS, 495, 502

Moster B. P., Somerville R. S., Maulbetsch C., van den Bosch F. C., Macciò A. V., Naab T., Oser L., 2010, ApJ, 710, 903

Nadler E. O. et al., 2021, Phys. Rev. Lett., 126, 091101

Navarro J. F., Frenk C. S., White S. D. M., 1997, ApJ, 490, 493

Nierenberg A. M. et al., 2017, MNRAS, 471, 2224

Ogiya G., Mori M., Miki Y., Boku T., Nakasato N., 2013, J. Phys.: Conf. Ser., 454, 012014

Ogiya G., van den Bosch F. C., Hahn O., Green S. B., Miller T. B., Burkert A., 2019, MNRAS, 485, 189

Ostriker J. P., Spitzer L. Jr, Chevalier R. A., 1972, ApJ, 176, L51

Parkinson H., Cole S., Helly J., 2008, MNRAS, 383, 557

Peñarrubia J., Navarro J. F., McConnachie A. W., 2008, ApJ, 673, 226 
Peñarrubia J., Benson A. J., Walker M. G., Gilmore G., McConnachie A. W., Mayer L., 2010, MNRAS, 406, 1290

Pillepich A. et al., 2018, MNRAS, 473, 4077

Plummer H. C., 1911, MNRAS, 71, 460

Robles V. H. et al., 2017, MNRAS, 472, 2945

Sawala T., Pihajoki P., Johansson P. H., Frenk C. S., Navarro J. F., Oman K. A., White S. D. M., 2017, MNRAS, 467, 4383

Somalwar J. J., Chang L. J., Mishra-Sharma S., Lisanti M., 2021, ApJ, 906, 57

Springel V. et al., 2008, MNRAS, 391, 1685

Stref M., Lavalle J., 2017, Phys. Rev. D, 95, 063003

Taylor J. E., Babul A., 2004, MNRAS, 348, 811

Tonini C., Lapi A., Salucci P., 2006, ApJ, 649, 591

van den Bosch F. C., Swaters R. A., 2001, MNRAS, 325, 1017

van den Bosch F. C., Ogiya G., Hahn O., Burkert A., 2018, MNRAS, 474, 3043

van den Bosch F. C., 2017, MNRAS, 468, 885

van den Bosch F. C., Ogiya G., 2018, MNRAS, 475, 4066

van den Bosch F. C., Jiang F., Campbell D., Behroozi P., 2016, MNRAS, 455, 158

Vegetti S., Koopmans L. V. E., Auger M. W., Treu T., Bolton A. S., 2014, MNRAS, 442, 2017

Wang H. Y., Jing Y. P., Mao S., Kang X., 2005, MNRAS, 364, 424

Wang Y., Yang X., Mo H. J., Li C., van den Bosch F. C., Fan Z., Chen X., 2008, MNRAS, 385, 1511

Wetzel A. R., 2011, MNRAS, 412, 49

Wetzel A. R., Hopkins P. F., Kim J.-h., Faucher-Giguère C.-A., Kereš D., Quataert E., 2016, ApJ, 827, L23

Yang X., van den Bosch F. C., Mo H. J., Mao S., Kang X., Weinmann S. M., Guo Y., Jing Y. P., 2006, MNRAS, 369, 1293

Zentner A. R., Berlind A. A., Bullock J. S., Kravtsov A. V., Wechsler R. H., 2005a, ApJ, 624, 505

Zentner A. R., Kravtsov A. V., Gnedin O. Y., Klypin A. A., 2005b, ApJ, 629, 219

Zhao D. H., Jing Y. P., Mo H. J., Börner G., 2009, ApJ, 707, 354

Zolotov A. et al., 2012, ApJ, 761, 71

\section{APPENDIX: IDEALIZED SIMULATIONS}

In order to verify that the Green et al. (2021) mass-loss model, which is given by equation (1), can be used to accurately describe subhalo evolution in a combined halo-disc host system, we run a set of idealized simulations that serve as the ground truth for comparison to our model predictions. We use the same procedure as used for the DASH simulations (Ogiya et al. 2019). In particular, all simulations are run using the $N$-body code OTOO + (Ogiya et al. 2013), which is a GPU-accelerated tree code. The simulations follow the evolution of a live $\mathrm{N}$-body subhalo (initially an NFW halo composed of $N=10^{6}$ particles) as it orbits within a static NFW host halo potential (with initial sub-to-host mass ratio of $m / M=10^{-3}$ ) for $36 \mathrm{Gyr}$.

In contrast to $D A S H$, the host system in our test suite of simulations is composed of an NFW halo with an embedded MN disc. The host halo has a concentration of $c_{\mathrm{vir}, \mathrm{h}}=10$ and the subhalo has an initial concentration of $c_{\mathrm{vir}, \mathrm{s}}=20$, which is consistent with a typical minor merger with a Milky Way-like host. For simplicity, we only consider orbits with orbital energy equal to that of a circular orbit at the virial radius of the host, which coincides with the peak of the orbital energy distribution of infalling subhaloes in cosmological simulations (e.g. Li et al. 2020).

When constructing our simulation suite, we vary several parameters, which control properties of the disc, the inclination of the initial subhalo orbit with respect to the disc, and the radius of orbital pericentre. The fraction of the host mass in the disc is set by $f_{M} \equiv$ $M_{\mathrm{d}} / M_{\text {vir }} \in[0.0,0.02,0.05,0.1]$. In order to preserve the total mass

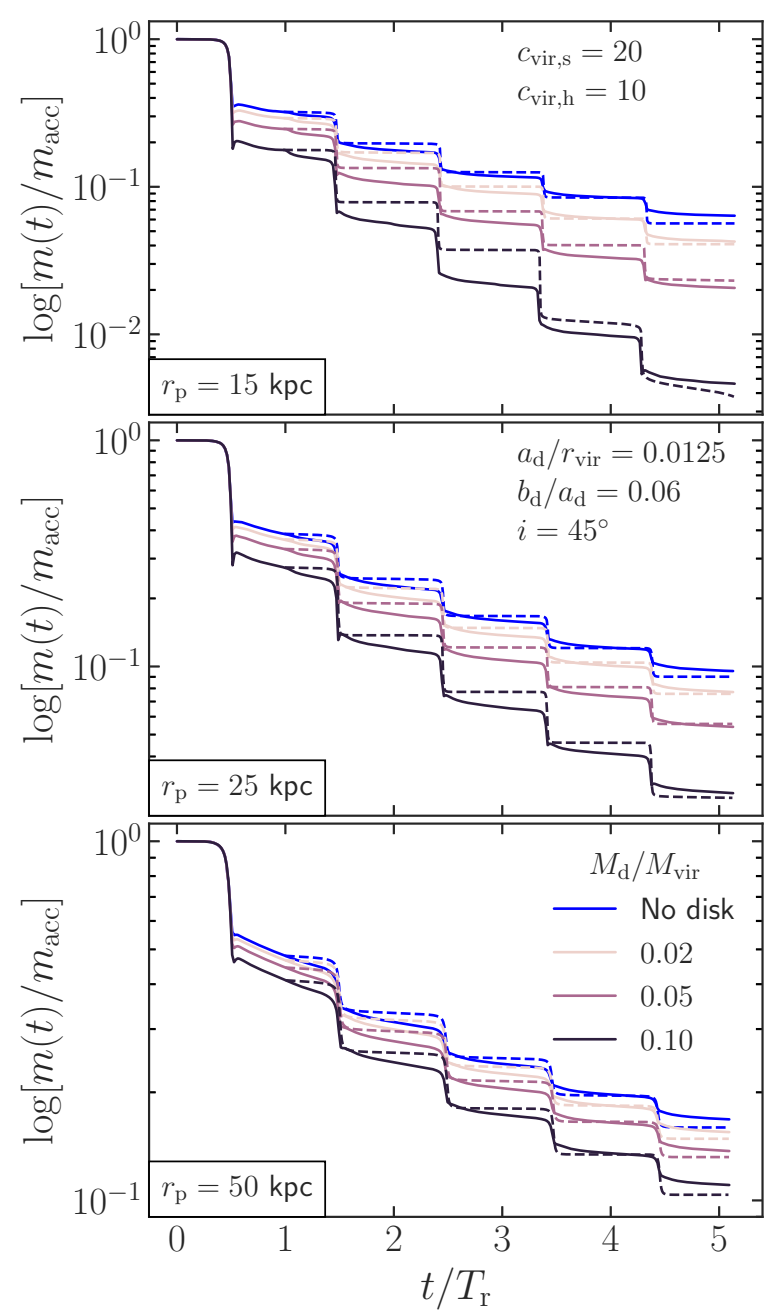

Figure A1. A comparison between the Green et al. (2021) mass-loss model predictions (dashed lines) and the $m(t) / m_{\text {acc }}$ trajectories of several simulated subhaloes (solid lines). The times are normalized by the radial orbital period, $T_{r}$. The host and subhalo concentrations, disc shape, orbital inclination, and orbital energy are held fixed. The model performs well over the full range of disc masses and down to small pericentric radii $\left(r_{\mathrm{p}}\right)$.

enclosed within $r_{\text {vir }}$, we multiply the host halo density profile by $1-$ $f_{M}$. The disc scale length is varied over $f_{a} \equiv a_{\mathrm{d}} / r_{\text {vir }} \in[0.007,0.0125$, $0.025]$, with $f_{a}=0.0125$ corresponding to $a_{\mathrm{d}} \approx 3.5 \mathrm{kpc}$ for the Milky Way-mass host, while the disc scale height is controlled by varying $b_{\mathrm{d}} / a_{\mathrm{d}} \in[0.02,0.06,0.2]$. We consider seven orbital inclinations with $i \in[0,15,30,45,60,75,90]$ degrees, where $i$ is the angle between the orbital plane and the disc (i.e. $i=0^{\circ}$ results in a subhalo that orbits in the plane of the disc). The final parameter is the orbital angular momentum, which we adjust such that the pericentric radius, $r_{\mathrm{p}}$, of the subhalo orbit in the no-disc configuration is equal to 15,25 , or $50 \mathrm{kpc}$.

Given the orbital energy, angular momentum, and inclination angle, we initialize the subhalo at its apocentre. Note that we use the same initial position and velocity regardless of the disc properties. Hence, the true $r_{\mathrm{p}}$ attained by the subhalo varies slightly with $f_{M}, f_{a}$, and $b_{\mathrm{d}} / a_{\mathrm{d}}$.

Thus, our test suite spans $f_{M}, f_{a}, b_{\mathrm{d}} / a_{\mathrm{d}}, i$, and $r_{\mathrm{p}}$. We follow the procedure laid out in Section 2.3.2 of Green et al. (2021) to 
generate mass-loss model predictions and make comparisons to the bound-mass trajectories, $m(t) / m_{\text {acc }}$, of the simulated subhaloes. The performance of the model is illustrated in Fig. A1, which compares the simulation and model results for the case of a fiducial MW-like disc shape and an inclined subhalo orbit with $i=45^{\circ}$. Clearly, the model accurately captures the subhalo mass evolution for all $M_{\mathrm{d}} / M_{\mathrm{vir}}$ and $r_{\mathrm{p}}$ considered. When averaged over the full test suite, we find that our model remains unbiased with relatively low scatter in the logresiduals (less than a factor of two larger than that of the halo-only case) for longer than a Hubble time, indicating that mass evolution error will be subdominant to the halo-to-halo variance. Hence, the mass-loss model successfully captures the additional loss of mass due to the presence of a disc, validating its use in SatGen for this study.

This paper has been typeset from a $\mathrm{T}_{\mathrm{E}} \mathrm{X} / \mathrm{L} \mathrm{AT} \mathrm{E} \mathrm{X}$ file prepared by the author. 